\title{
In situ Gastric pH Imaging with Hydrogel Capsule Isolated Paramagnetic Metallo-albumin Complexes
}

Yiting $\mathrm{Xu},{ }^{[1]}$ Yanxia Yang, ${ }^{[1]}$ Zhiwei Yin, ${ }^{[1]}$ Xinqi Cai, ${ }^{[1]}$ Xin Xia, ${ }^{[1]}$ Michael J Donovan, ${ }^{[1]}$ Long Chen, ${ }^{[2]}$ Zhuo Chen ${ }^{[1]}, *$ and Weihong Tan ${ }^{[1,3]}$

[1] Molecular Science and Biomedicine Laboratory, State Key Laboratory of Chemo/Biosensing and Chemometrics, Hunan Provincial Key Laboratory of Biomacromolecular Chemical Biology, College of Chemistry and Chemical Engineering, College of Life Sciences, Aptamer Engineering Center of Hunan Province, Hunan University, Changsha, Hunan, 410082, China, E-mail: zhuochen@hnu.edu.cn

[2] Faculty of Science and Technology, University of Macau, Avenida da Universidade, Taipa, 999078, Macau

[3] Institute of Cancer and Basic Medicine (IBMC), Chinese Academy of Sciences; The Cancer Hospital of the University of Chinese Academy of Sciences, Hangzhou, Zhejiang, 310022, China

Correspondence and requests for materials should be addressed to Zhuo Chen (email: zhuochen@hnu.edu.cn). 


\section{MATERIALS AND METHODS}

Reagents. Human Serum Albumin was purchased from Beijing Biosharp Technologies Corporation (Beijing, China). Manganese standard solution was obtained from Aladdin Industrial Corporation (Shanghai, China). 3-morpholinopropane sulfonic acid (MOPS) was supplied by Adamas Reagent Corporation (Shanghai, China). Gastric pepsin, and Mucin type II were purchased from Sigma-Aldrich (St Louis, MO, USA). Agarose was obtained from Thermo Fisher Scientific Corporation (Shanghai, China). Vonoprazan Fumarate was purchased from Aladdin Industrial Corporation (Shanghai, China). All reagents were used directly without further purification.

pH reversibility of HSA-Mn system. HSA-Mn solutions ( $0.2 \mathrm{mM} \mathrm{Mn(II),} 1.4 \mathrm{mM} \mathrm{HSA}$, dd $\mathrm{H}_{2} \mathrm{O}$ solved) were prepare and the $\mathrm{pH}$ were adjusted by $1 \mathrm{M} \mathrm{HCl}$ solution and $1 \mathrm{M} \mathrm{NaOH}$ solution.

Agarose hydrogel capsule. Agarose were weighted at $2 \% / 3 \% / 4 \%$ (weight $\%$ to $\mathrm{ddH}_{2} \mathrm{O}$ ) in flasks and heated to $100{ }^{\circ} \mathrm{C}$ by a microwave oven. The heated gel solution was pour into a tailor-made mold and then cooled down to room temperature. After taking apart the mold, the completely molding hydrogel capsules were washed by $\mathrm{ddH}_{2} \mathrm{O}$ for at least 6 hours to completely remove the unbound gel monomer. Prepared agarose hydrogel capsules were protected with preservative film and set at $4{ }^{\circ} \mathrm{C}$ for further use.

Effective encapsulation of the HSA and Mn(II) ion. In order to effective encapsulate the HSA-Mn adduct with hydrogel capsule, we further optimizing the gel concentration and HSA concentration. When testing gel concentration, a mixed solution of $0.2 \mathrm{mM} \mathrm{Mn}(\mathrm{II})$ and $1.0 \mathrm{mM} \mathrm{HSA}$ solved in $\mathrm{ddH}_{2} \mathrm{O}$ was used as S-inner. The mixed solutions were added to the hydrogel capsule of different concentration: $2 \mathrm{wt} \%, 3$ $\mathrm{wt} \%, 4 \mathrm{wt} \%$. When testing whether increased concentration of HSA could constraint Mn(II) to decrease the leakage from S-inner, we changed the concentration of HSA at 0, 0.6, 1.0, 1.4, $1.8 \mathrm{mM}$ where $\mathrm{Mn}$ (II) concentration was set as $0.2 \mathrm{mM}$. HSA-Mn solutions were added to the hydrogel capsules and immersed in a MOPS buffer, $\mathrm{pH} 7$, which temperature was maintained at $37^{\circ} \mathrm{C}$ by using a water bath. At each time point $(0,5,10,30,60 \mathrm{~min})$, minimum samples were taken out for $\mathrm{T}_{1}$ measurement and HSA and $\mathrm{Mn}$ (II) quantification. Spectrum absorbance at $280 \mathrm{~nm}$ of HSA was used to quantify its concentration, while $\mathrm{Mn}$ (II) concentration were quantified through inductively coupled plasma mass spectrometry (ICP-MS, Aglient, 8900).

\section{MRI of the HSA-Mn hydrogel capsule in vitro.}

10 and $50 \mathrm{~mL} \mathrm{SGF}, \mathrm{pH}$ 1-7, were prepared. The HSA-Mn hydrogel capsules were immersed into different pH SGF and imaging was taken at 0, 5, 10, 30, 60 minutes with a 7 T MRI machine (Bruker PharmaScan $70 / 16$ US). To better understand the $\mathrm{pH}$ response mechanism of the HSA-Mn hydrogel capsule which based on the fast diffusion equilibrium of $\mathrm{H}^{+}$from S-outer to S-inner, Fick's second law ${ }^{1}$ (3) and Stocks-Einstein equation ${ }^{2}$ (4) have been applied. In Fick's second law equation, $C$ is the concentration of diffusion particle, $t$ represents diffusion time, $x$ is diffusion distance, $D$ is diffusion coefficient, while Stocks-Einstein equation further illuminate the relationship between $D$ and temperature $(T)$, viscosity of diffusion medium $(\eta)$ and hydrodynamic diameter of particles $(d(H))$.

$\partial C /(\partial t)=\partial / \partial x(D \partial C / \partial x)$ 
Once immersed in the acidic solution, the large concentration gradient drives the rapid diffusion of relevant molecules and ions to the gel. The $D$ is inversely proportional to hydrodynamic diameter $(\mathrm{d}(\mathrm{H}))$ and the viscosity of diffusion medium $(\eta)$. The latter is related to the pore size of the gel $\left(d_{\text {pore }}\right)$, which is proportional to gel concentration ${ }^{3}$. The effective diffusion coefficient of HSA ( $\left.D_{H S A}\right)$ was much lower than $D_{H}$ because of the great $d(H)$ difference between them ( 1000 fold). As for $\mathrm{Mn}(\mathrm{II})$ and $\mathrm{H}^{+}$, the difference of $d(H)$ is not that significant, but the results in Figure $3 b$ showed that the capsule sensor still worked. One possible reason was the binding of $\mathrm{Mn}$ (II) to HSA lower the effective diffusion coefficient of $\mathrm{Mn}$ (II) $\left(D_{\mathrm{Mn}(I I)}\right)$, which resulted from the increased hydrodynamic diameter and also the limited mobility of $\mathrm{Mn}(\mathrm{II})$ after binding (Figure S2f).

Pore size of gel. For further investigate the change of pore size and distribution in hydrogel capsules after immersed in different pH SGF, gel cylinder (diameter was $9.1 \mathrm{~mm}$ ) were cut into spices and frize-dried after emersion in $\mathrm{pH}$ 1-7 SGF for 1 hour $\left(37^{\circ} \mathrm{C}\right)$. Pore size was detected through $\mathrm{N}_{2}$ adsorption experiments (BET).

Animals and diet. Pasteurella-free New Zealand White rabbits (5 7 weeks) were purchased from Hunan Taiping biotechnology Co. All animals received care compliance with the guidelines in the Guide for the Care and Use of Laboratory Animals, and the procedures of animal experiments were ensured by the Institutional Animal Care and Use Committee of Hunan University. The experimental methods were carried out following governmental and international guidelines on animal experimentation, strictly. Best efforts were made to use least animals and relieve the pain during experiments according to the request of Biosafety and Animal Ethics. All rabbits were fed with artificial rabbit food and $\mathrm{ddH}_{2} \mathrm{O}$ during the whole experiment process.

MRI of gastric acid with HSA-Mn hydrogel capsule.

L-size capsule ( $\phi 9.1 \times 25 \mathrm{~mm}): 12 \mathrm{New}$ Zealand White rabbits $(\sim 500 \mathrm{~g})$ were ramdonly divided to two groups: normal and abnormal gastric $\mathrm{pH}$ group. Abnormal group was revealed by a satuated $\mathrm{NaHCO}_{3}$ solution. After anesthesia, the L-size capsules were buried into the stomachs of rabbits through a open laparotomy method. $\mathrm{T}_{1} \mathrm{MRI}$ were taken every 5 minutes.

The HSA-Mn hydrogel capsules (large size capsule: $\phi 9.1 \times 25 \mathrm{~mm}$ ) were first applied in vivo through an open laparotomy method (S5a). Results in Figure S5b indicated that the capsule distinctly located in the stomach and the bright $\mathrm{T}_{1}$ signal was confined within the cavity of capsule. As the time went by, this $\mathrm{T}_{1}$ signal clearly darkened in normal gastric $\mathrm{pH}$, while maintaining brightness in the abnormal $\mathrm{pH}$ group. The normalized intensity of S-inner/Head $(\mathrm{S} / \mathrm{H})$ results represented in Figure S6c-d indicated that the two groups took obviously different variation trends. Taking 30 minutes as the detection time point, the result shown in Figure S6e indicated the quantitative result of the normalized S/H intensity was statistical significant between normal and abnormal gastric $\mathrm{pH}$ group $\left(0.70 \pm 0.21\right.$ vs $\left.1.07 \pm 0.05 ;{ }^{* *} \mathrm{P}<0.01\right)$. These results amply demonstrated the feasibility of the HSA-Mn hydrogel capsule for in situ MRI monitoring abnormal gastric $\mathrm{pH}$.

Safety assessment. After orally administrated S-size capsules for 48 hours, the main organs of rabbits were harvested for htoxylin and eosin stainning (H\&E). Besides, the excrement of rabbits were collected 
before and after experiment to investigate the gastrointestinal function.

To further verify the biosafety of the paramagnetic matallo-protein complexes, the S-inner was oral administrated to rabbits for 7 days. After 7 days, the rabbits were sacrificed and the major organs were taken for Hematoxylin and eosin (H\&E) staining. The results shown in Figure S10 indicated no significant differences when compared with the control group $\left(\mathrm{ddH}_{2} \mathrm{O}\right.$ fed), which suggested negligible toxicity of the $\mathrm{S}$-inner. It should be mentioned that, beneficial from the signal confinement ability of the capsule, the total quantity of $\mathrm{Mn}^{2+}$ intake was no more than $1.1 \mathrm{mg}$ (1.1 mg for one L-capsule). That's roughly $1 / 2$ of recommended total daily intake of $\mathrm{Mn}^{2+}$ for human ${ }^{4}$. Besides, body weight of all individuals without big fluctuation during the experiment also verified the biosafety of the capsules (Figure S11).

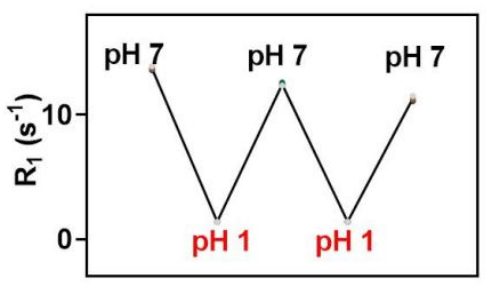

Figure S1. pH reversibility of HSA-Mn system $\left(1.5 \mathrm{~T}, 37^{\circ} \mathrm{C}\right)$.
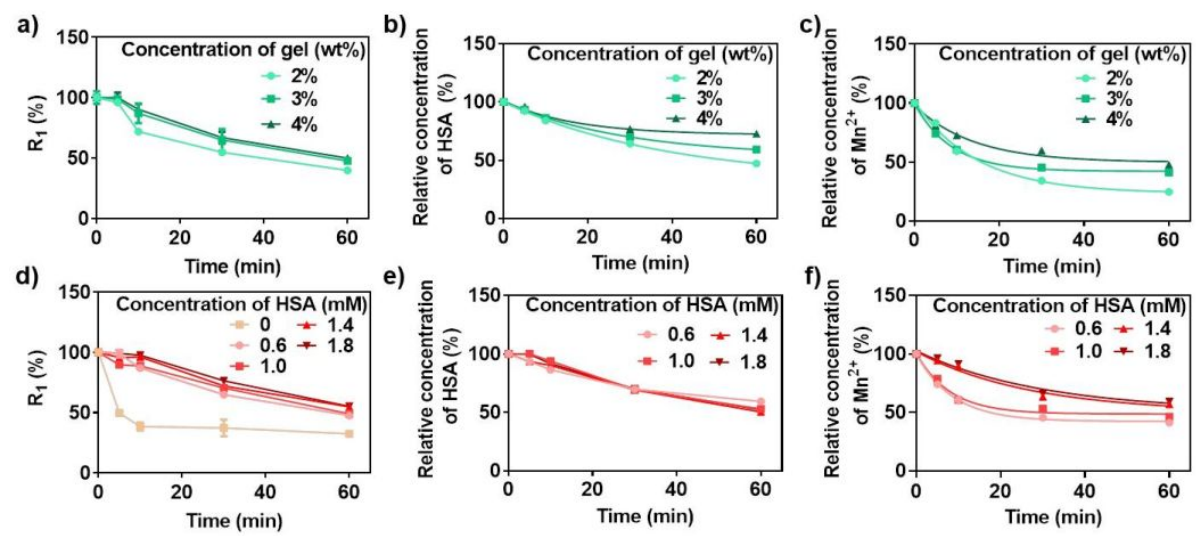

Figure S2. Effective encapsulation of the HSA and Mn(II) by optimizing the a) b) c): gel concentration and d) e) f): HSA ratio.

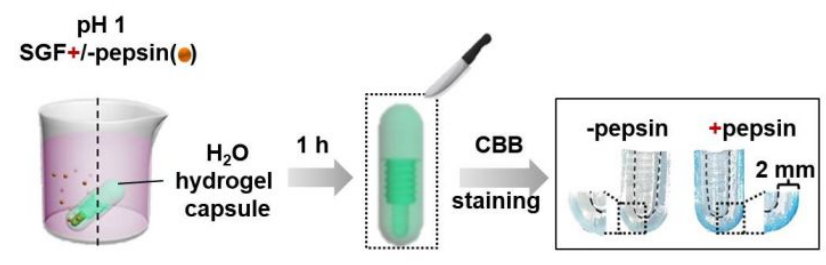

Figure S3. Pepsin penetration to the capsule gel layer after immersed in SGF with or without pepsin in $37^{\circ} \mathrm{C}$ water bath for 1 hours. 
a)

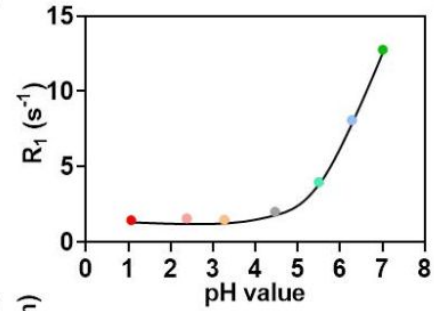

c)

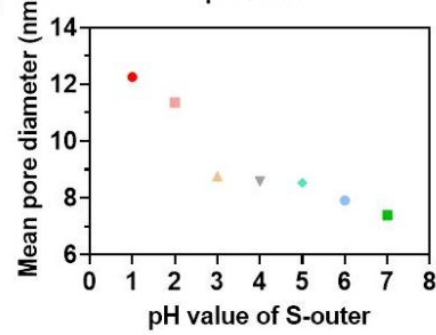

b)

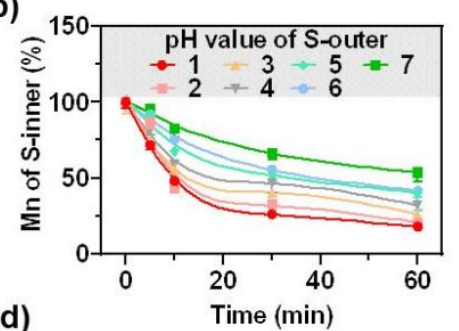

d)

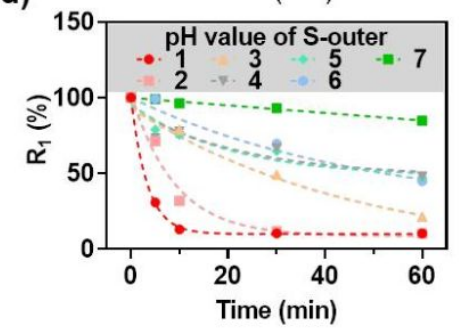

Figure S4. a) The mean pore diameter of gel measured by BET method after immersed in different pH SGF for 1 hour. b). Concentration of encapsulated $\mathrm{Mn}(\mathrm{II})$ over time. c) $\mathrm{R}_{1}$ of HSA-Mn system in $\mathrm{pH}$ 1-7. non-linear fitting function was calculated as: $y=$ $1.48+15.96 /\left(1+10^{\wedge}\left((6.48-X)^{*} 0.73\right)\right), R^{2}=0.999$. d) Theoretical $R_{1}$ of $S$-inner based on non-linear fitting function in a) and $p H$ of $S$-inner in Figure 3a).

a)

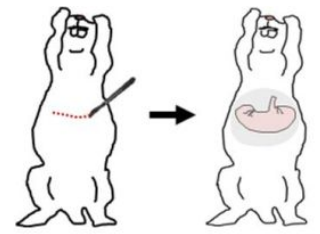
Time (min)

b)

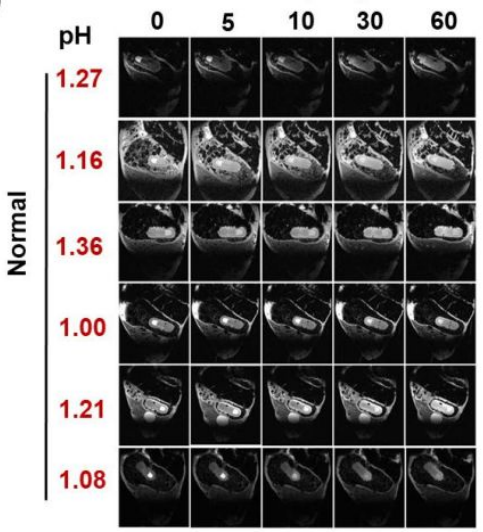

c)

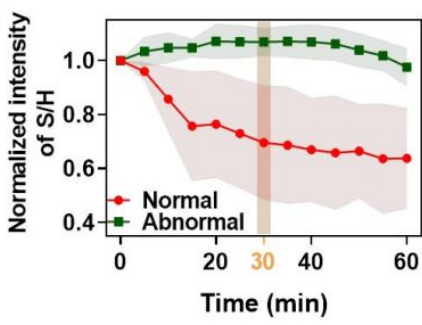

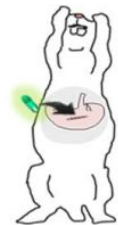

60 d)
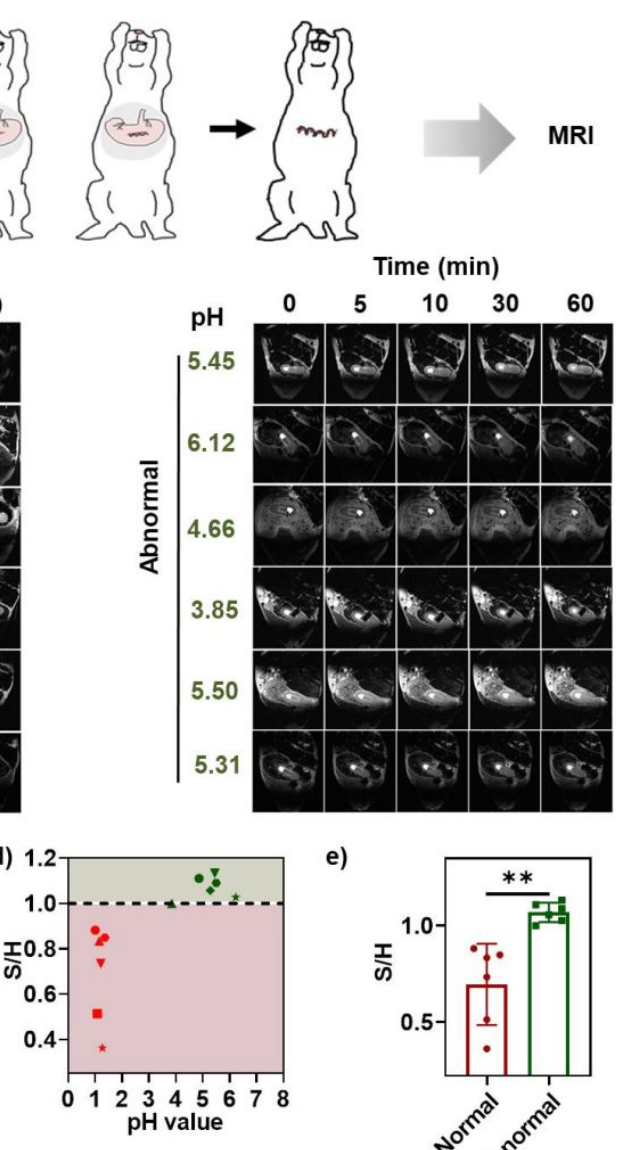

MRI

e)

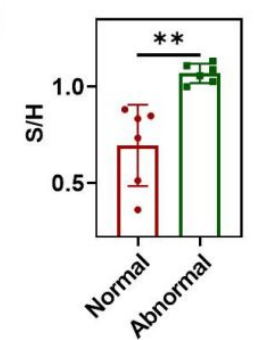

Figure S5. In situ MRI of gastric $\mathrm{pH}$ by HSA-Mn hydrogel capsule (L-size capsule: $\phi \mathbf{9 . 1} \times \mathbf{2 5} \mathrm{mm}$ ). a) Illustration of the gastric $\mathrm{pH}$ monitoring process with L-capsules. The capsules were buried into the rabbits' stomach through open laparotomy. b) MRI of capsule at different $\mathrm{pH}$ gastric $\mathrm{pH}$ at time points. c) Quantification results of b). e) Statistical assessment of b) at 30 minutes. $\left({ }^{* *} \mathrm{P}<0.01\right)$. 

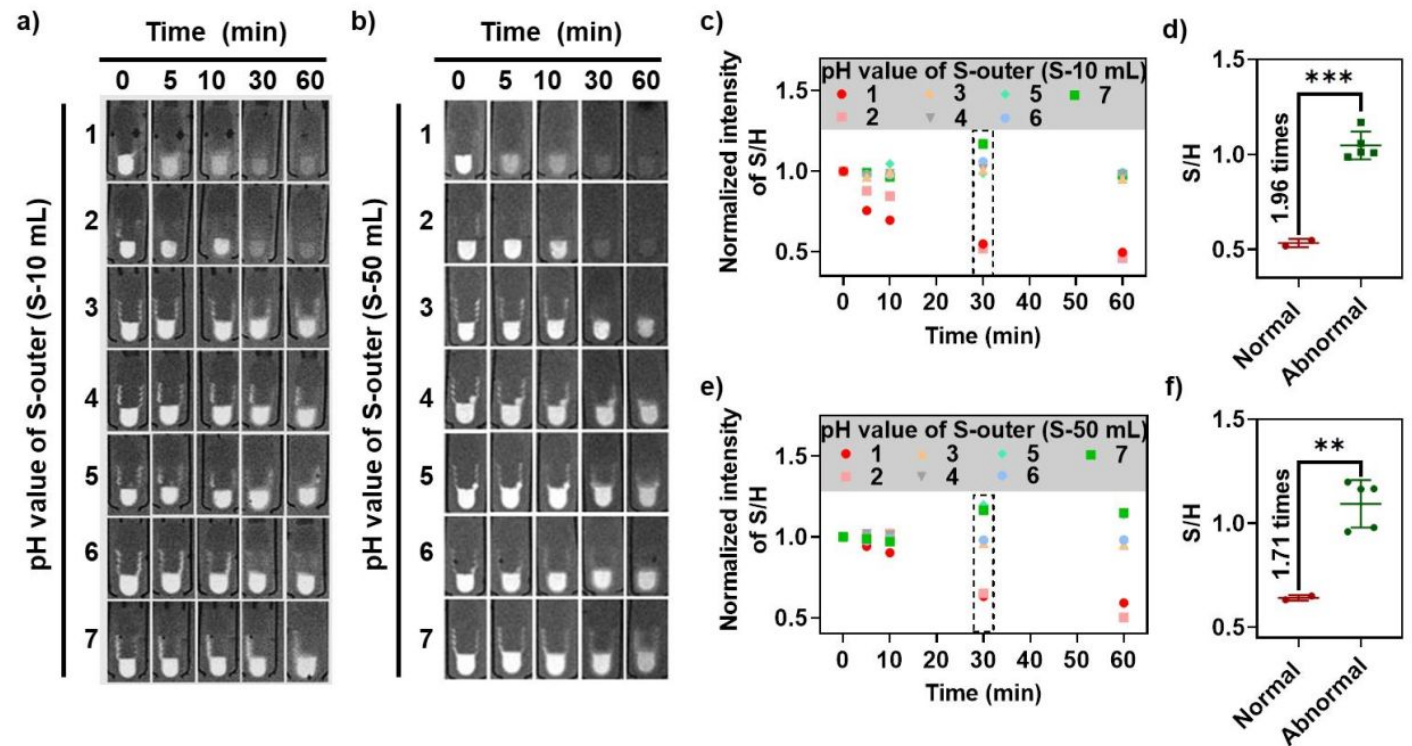

Figure S6. MRI of HSA-Mn hydrogel capsule (S size: $\phi 5.6 \times 18 \mathrm{~mm}$ ) in SGF. a) Demonstration of imaging experiment. MRI of capsule immersed in different volume and pH SGF. 7 T. a) $10 \mathrm{~mL}$ and b) $50 \mathrm{~mL}$. c) e). Quantification results of a) and b). d), f) Statistical assessment of $\mathrm{C}$ ) and e) at $30 \mathrm{~min} .\left({ }^{* * *} \mathrm{P}<0.001 .{ }^{* *} \mathrm{P}<0.01\right) .\left(7 \mathrm{~T}, 37^{\circ} \mathrm{C}\right)$.
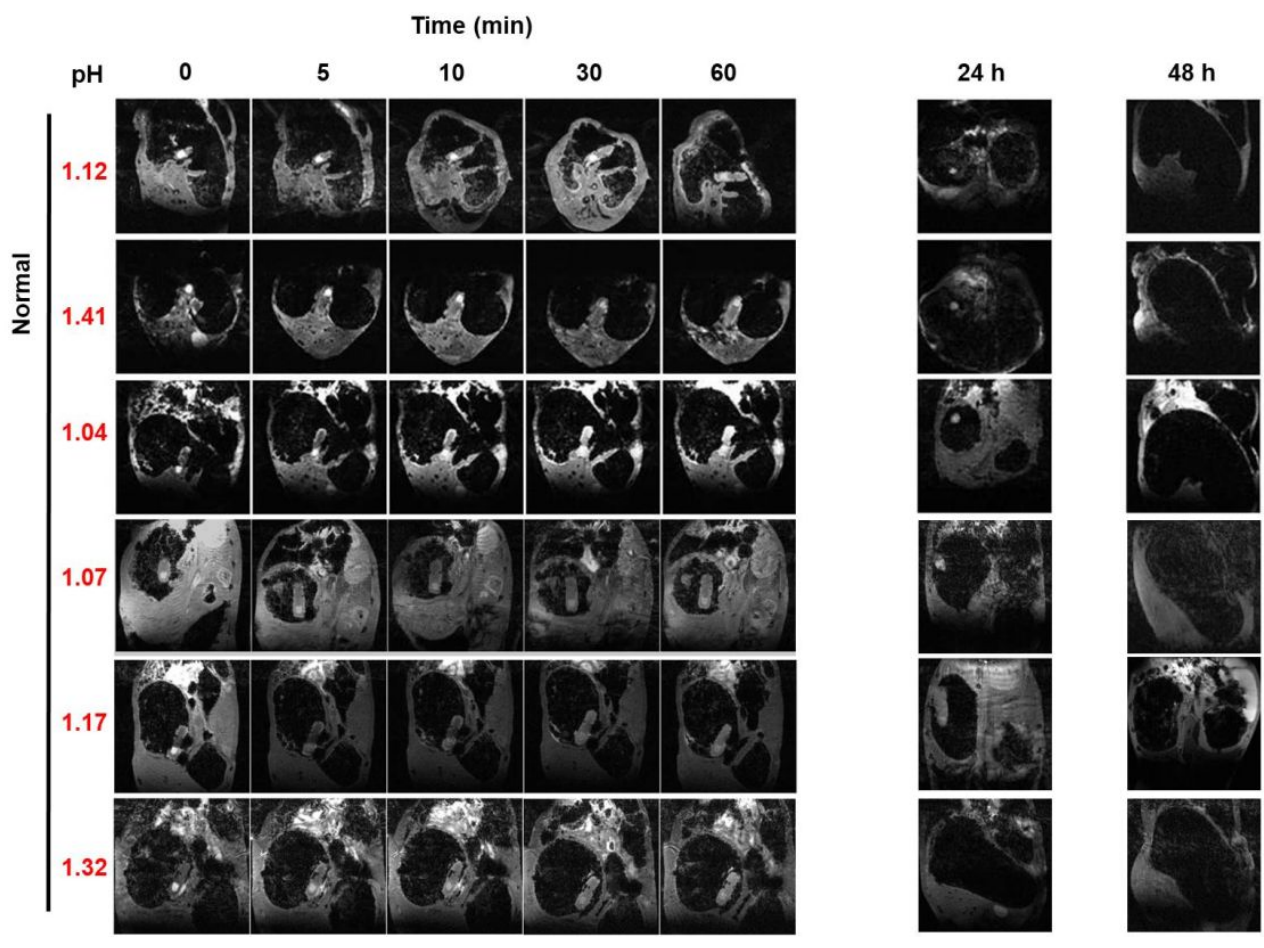

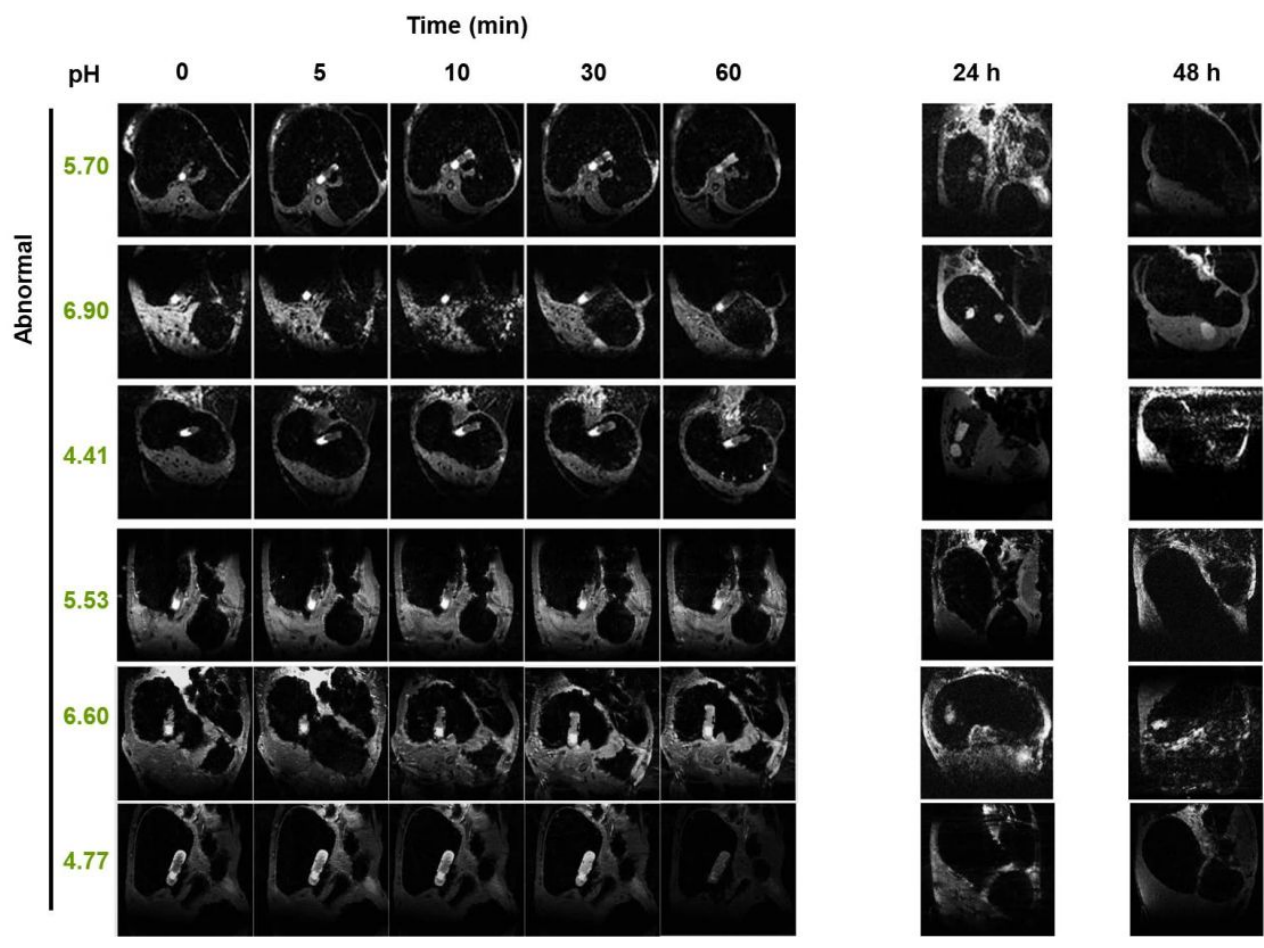

Figure S7. MRI of HSA-Mn hydrogel capsule in vivo. (S size: $\phi 5.6 \times 18 \mathrm{~mm})$.

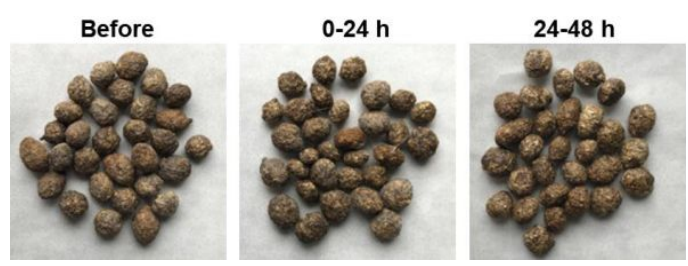

Figure S8. Safety assessment. Excrement pictures of rabbits before and after oral administration of HSA-Mn hydrogel capsules.

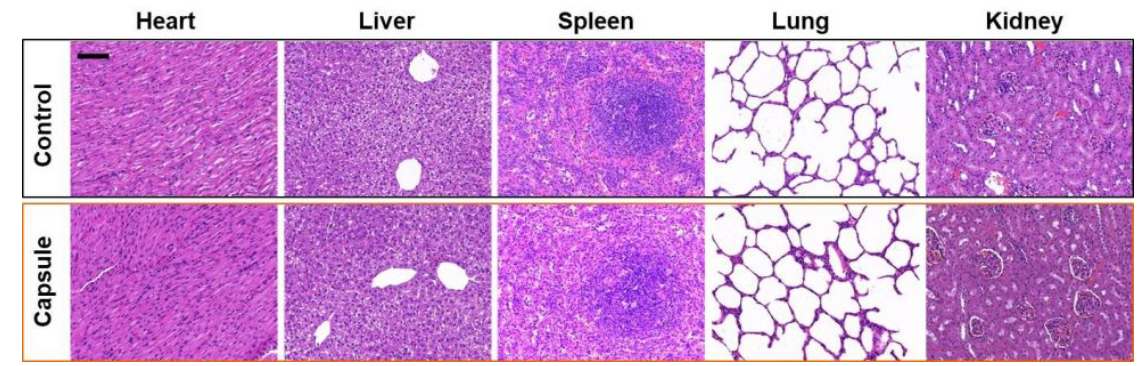

Figure S9. H\&E staining of the main organs 48 hours after oral administrating of HSA-Mn hydrogel capsules. Scale bar is $200 \mu \mathrm{m}$ 

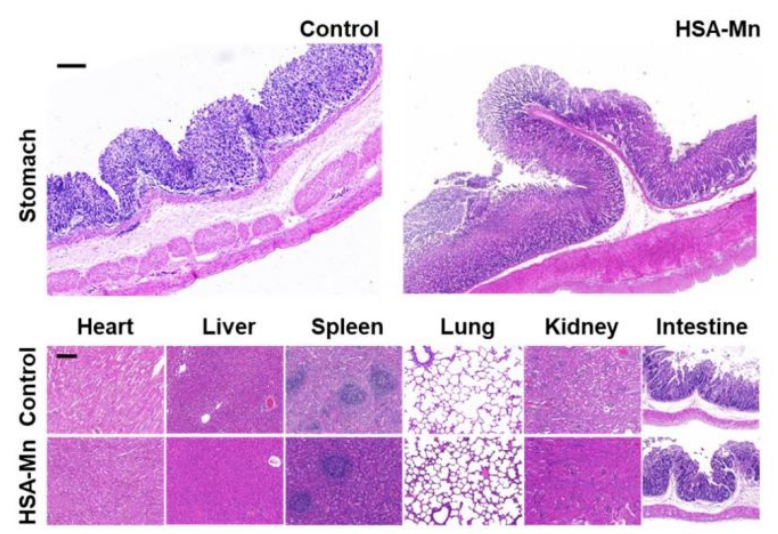

Figure S10. H\&E staining of the main organs after oral administration of HSA-Mn solution for 7 days. Scale bar is $200 \mu \mathrm{m}$.

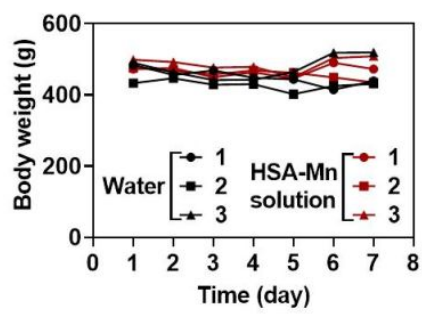

Figure S11. Body weight of the rabbits with HSA-Mn solution or $\mathrm{ddH}_{2} \mathrm{O}$ fed for 7 days.

\section{References}

1. Labille, J.; Rouge, N. F. Buffle, J., Local and Average Diffusion of Nanosolutes in Agarose Gel: The Effect of the Gel Solution Interface Structure. Langmuir 2007, 23 (4), 2083-2090.

2. Amsden, B.; Solute Diffusion within Hydrogels. Mechanisms and Models. Macromolecules 1998, 31 (23), 8382-8395.

3. Gutenwik, J.; Nilsson, B.; Axelsson, A., Effect of hindered diffusion on the adsorption of proteins in agarose gel using a pore model. J. Chromatogr. A 2004, 1048 (2), 161-172.

4. Institute of Medicine (US) Panel on Micronutrients, Dietary Reference Intakes for Vitamin A, Vitamin K, Arsenic, Boron, Chromium, Copper, lodine, Iron, Manganese, Molybdenum, Nickel, Silicon, Vanadium, and Zinc. Washington (DC): National Academies Press (US) 2001. 